\title{
Six Month Trajectories of COVID-19 Experiences and Associated Stress, Anxiety, Depression, and Impairment in American Adults
}

\author{
Matthew W. Gallagher ${ }^{1,2,3}$ (1) $\cdot$ Lia J. Smith ${ }^{1} \cdot$ Angela L. Richardson $^{1} \cdot$ Laura J. Long $^{1}$ \\ Accepted: 1 November 2021 / Published online: 16 November 2021 \\ (c) The Author(s), under exclusive licence to Springer Science+Business Media, LLC, part of Springer Nature 2021
}

\begin{abstract}
Background The COVID-19 pandemic has had a profound impact on health and well-being worldwide. There is increasing research seeking to better understand the psychological impact of COVID-19 experiences. However, this research has largely been limited in size and scope.

Methods The present study examined longitudinal trajectories of COVID-19 experiences on COVID-19 related stress, anxiety, depression, and functional impairment in a convenience sample of 788 American adults recruited through MTURK. Data was collected across four waves between March and October 2020.

Results COVID-19 experiences were consistently associated with higher odds of probable anxiety and depression diagnoses. COVID-19 related stress also predicted large proportions of variance in anxiety, depression, and functional impairment in latent variable analyses. Overtime, the results indicated that while anxiety and depression decreased, functional impairment remained stable.

Conclusions These findings highlight the emotional toll of the COVID-19 pandemic over time. Decreases in COVID-19 related stress, anxiety, and depression over time may reflect resiliency among respondents. Importantly, these results underscore the continued need for mental health services as associations between COVID-19 and functional impairment remained consistent over time.
\end{abstract}

Keywords COVID-19 $\cdot$ Coronavirus $\cdot$ Stress $\cdot$ Anxiety $\cdot$ Depression $\cdot$ Impairment $\cdot$ Longitudinal

The ongoing 2019 novel coronavirus (COVID-19) pandemic has profoundly impacted communities across the globe. As of October 2021, upwards of 244 million infections and 4 million deaths have been connected to COVID-19 worldwide (Johns Hopkins University, 2021). Since the beginning of the pandemic, there has been concern that stress associated with COVID-19 may have deleterious impacts overtime. Indeed, ongoing uncertainty, dramatic behavioral shifts, and vaccine concerns related to the pandemic may uniquely impact mental health (Gruber et al., 2020;

Matthew W. Gallagher

mwgallagher@uh.edu

1 Department of Psychology, University of Houston, Houston, TX, USA

2 Texas Institute for Measurement Evaluation and Statistics (TIMES), Houston, TX, USA

3 Department of Psychology, Texas Institute for Measurement, Evaluation, and Statistics, University of Houston, 4349 Martin Luther King Blvd, Rm 373, Houston, USA
Karlsson et al., 2021; Pfefferbaum \& North, 2020; Wang et al., 2020a).

Numerous challenges associated with the COVID-19 global health pandemic may contribute to increased levels of perceived stress. Perceived stress reflects the degree to which individuals appraise their life as stressful by evaluating how unpredictable, uncontrollable, and overloaded one perceives their life to be (Cohen et al., 1983; Lee, 2012) and has consistently been associated with reductions in mental and physical health outcomes (e.g., Lee, 2012; Redmond et al., 2013). Emerging cross-sectional research examining perceived stress associated with the COVID-19 pandemic has documented a higher likelihood of perceived stress associated with particular COVID-19 experiences (e.g., confirmed COVID-19 diagnosis; Gallagher et al., 2020), social distancing procedures (Badola et al., 2021), pandemic-related media coverage (Bendau et al., 2021), and inconsistent pandemic-related policies (Pedrozo-Pupo et al., 2020). Direct care of COVID-19 patients has also been cross-sectionally associated with increased perceived 
stress among healthcare workers (Babore et al., 2020; Das et al., 2020). Preliminary cross-sectional research suggests that perceived stress related to COVID-19 has been associated with a range of maladaptive (e.g., substance use, denial, behavioral disengagement) and adaptive (e.g., use of emotional support, humor, religion) coping strategies among individuals with self-reported disabilities and chronic conditions (Umucu \& Lee, 2020). In sum, while some associations between the COVID-19 pandemic and perceived stress have been documented, increased understanding is needed regarding the course of these associations over time.

Emerging research has also shown that COVID-19 experiences are associated with higher likelihood of anxiety and depression symptoms (Gallagher et al., 2020; Hossain et al., 2020). A retrospective study of patients with COVID-19 found that in the 6 months following diagnosis, $8.63 \%$ were diagnosed with an anxiety, mood, or psychotic disorder for the first time (Taquet et al., 2021). These initial findings are to be somewhat expected, given the role of stress in the etiology of these disorders (Chrousos, 2009; de Kloet et al., 2005) and ongoing pandemic-related loss, lack of control, risk of disease, and financial impact. Theoretically, chronic feelings of anxiety and depression following acute experiences of stress related to the COVID-19 pandemic may further deplete our resources, as is proposed by the allostatic load model (McEwen, 2005). Indeed, preliminary evidence suggests that mental health symptomatology may be associated with more severe COVID-19 progression (Taquet et al., 2021; Yao et al., 2020). Overall, while this emerging body of work continues to document psychiatric impacts of the COVID-19 pandemic, functional impacts over the course of the pandemic are less clear.

While less documented in emerging research, COVID19 experiences are likely to influence both psychiatric and functional domains over the course of the pandemic. Preliminary cross-sectional findings in this area suggest that COVID-19 experiences and associated stress are predictive of functional impairment (Gallagher et al., 2020). One study examining bereaved persons following the death of a loved one due to COVID-19, found that $63.2 \%$ of the sample exhibited clinically significant levels of functional impairment. Functional impairment was positively associated with being diagnosed with COVID-19 and the odds of functional impairment significantly increased for bereaved persons exhibiting increased symptoms of posttraumatic stress, separation distress, and dysfunctional grief (Breen et al., 2021). Past work across other medical illnesses (e.g., diabetes, sleep disturbance) underscore that increased disease severity is associated with greater functional impairment (Littlefield et al., 1990; Nyer et al., 2013) and that this association may be exacerbated by concurrent mental health symptoms (e.g., anxiety and depression; Kim et al., 2000).
Therefore, it is plausible that COVID-19 experiences may also impact functional impairment over time.

Despite the emerging body of cross-sectional research examining psychiatric and functional impacts of the COVID-19 pandemic, increased longitudinal examinations of these relationships are needed. Available longitudinal surveys have indicated increased in mental health difficulties during the early acute phase of the pandemic, when compared to pre-pandemic levels in both adult (e.g., Daly et al., 2020; Pierce et al., 2020), college (Li et al., 2021), and adolescent (De France et al., 2021) samples. Longitudinal surveys examining mental health symptoms during the initial COVID-19 outbreak and subsequent timepoints, however, have had some mixed results. For example, Wang et al. (2020b) found reductions in posttraumatic stress symptoms, but no significant changes in stress, anxiety, or depression over the course of 1 month among adults in China. In contrast, researchers in England found symptoms of anxiety and depression to decrease over time (Fancourt et al., 2021). Studies conducted in England and Germany, however, found a reduction in anxiety and depression over the course of the pandemic (Bendau et al., 2021; Fancourt et al., 2021). While these results provide a developing understanding of the psychological impact of the COVID-19 pandemic, they are not necessarily generalizable to the United States population. Examinations of how perceived stress, anxiety, depression, and functional impairment may be related to COVID-19 experiences overtime could provide a better understanding of the course of the pandemic and critical time periods for prevention and intervention services. This longitudinal work is critical given the impact of serious medical illnesses, such as COVID-19, on mental health outcomes (Moos \& Schaefer, 1984; Turner \& Baker, 2010), burden of care for COVID-19 patients (Hickman \& Douglas, 2010), and amount of loss experienced over the course of the pandemic thus far (Eisma et al., 2020; Gallagher et al., 2020; Ott, 2003). The current study examined longitudinal trajectories of COVID-19 experiences and related stress on anxiety, depression, and functional impairment over time. We hypothesized that those who received a medical confirmation of COVID-19 or knew someone who died from COVID-19 would report greater anxiety, depression, and functional impairment over time.

\section{Methods}

\section{Participants}

Participants included adults in the United States that were recruited through Amazon Mechanical Turk (MTURK) during March 2020. Participants were eligible for inclusion if they: (1) were 18 years or older, (2) were living in the United 
States, (3) had completed at least 100 Human Intelligence Tasks (HITS), and (4) had a $95 \%$ or greater HIT approval rate. Participants were excluded if they answered less than 3 out of 4 validity questions correctly (e.g., "Choose '5' for this question"), reported inconsistent age information across different timepoints, or gave invalid responses to short answer questions about their experiences during the COVID19 pandemic. These quality control procedures were selected based on past reviews of best practices to maximize data quality when collecting data using the MTURK platform (Thomas \& Clifford, 2017). The present study had a targeted sample size of 500 based on an a-priori power analysis indicating that this sample size would provide power greater than 0.80 to detect small to medium associations $(r s \geq 0.20)$ at alpha of 0.01 .

The final sample consisted of 788 participants with an average age of 37.96 (SD $=11.81$; range 18-73). Participants mostly identified as White $(70.3 \%)$, with the remaining identifying as African American/Black (13.5\%), Hispanic/ Latin@ (5.1\%), Asian/Pacific Islander (4.6\%), Native American $(2.8 \%)$, or multiracial/other $(3.8 \%)$. Most of the participants identified as male $(58.1 \%)$, heterosexual $(85.7 \%)$, married $(52.7 \%)$, having children $(57.4 \%)$, employed $(77.8 \%)$, having health insurance $(79.1 \%)$, and had a bachelor's degree or higher level of education (43.5\%). Participants reported an average household size of 3 individuals (range 1-12) and, among those that endorsed having children, reported having 2 children on average (range 1-10). The average household income was $\$ 69,889$ (range $\$ 1000-\$ 100,000$ ).

Out of the final sample of participants, $18.5 \%$ reported receiving a confirmed diagnosis of COVID-19, and $26.1 \%$ reported that somebody they knew had died of COVID-19 across any of the four timepoints. Further, $27.0 \%$ of participants reported that they were at greater risk of contracting COVID-19 (due to pre-existing conditions, being immunocompromised, age, etc.) and $58.5 \%$ of participants endorsed having immediate family members at greater risk for contracting COVID-19. Among those that were employed, $41.6 \%$ reported that they were unable to go to work due to COVID-19 social distancing procedures. Approximately $81.2 \%$ of participants reported that they had been at least somewhat financially impacted by the pandemic.

\section{Procedures}

Study participants were recruited through MTURK, an online crowdsourcing marketplace. This platform is commonly used in the context of academic research given that it has been shown to be a reliable and valid method of data collection (Thomas \& Clifford, 2017). HITS posted to the platform were described as part of a longitudinal research study examining mental health during the COVID-19 pandemic. Data was collected across 4 timepoints occurring between March 23, 2020 and May, 28, 2020 for timepoint
1, May 7, 2020 and June 29, 2020 for timepoint 2, June 8, 2020 and August 10, 2020 for timepoint 3, and September 10, 2020 and October 24, 2020 for timepoint 4. Participants were invited to complete follow-up surveys approximately 3-6 weeks after being invited to complete the HIT for each previous timepoint, and they could receive up to three emails reminding them to complete each HIT. Though 858 participants completed the initial survey at timepoint 1 , those who failed to answer 3 out of the 4 validity questions correctly, showed suspicious geolocations, reported conflicting ages across different timepoints, or provided invalid responses to short answer questions about their coronavirus experiences were excluded from the final analysis $(n=70)$. Across the course of longitudinal data collection, $78 \%$ of time 1 participants $(n=788)$ responded at time $2(n=612), 71 \%$ at time $3(n=558)$, and $60.79 \%(n=479)$ at time 4 . All individuals who met initial eligibility criteria and participated at timepoint 1 could complete the survey at any timepoint. The study was approved by the Institutional Review Board at the University of Houston.

\section{Materials}

\section{COVID-19 Experiences}

The survey included questions that were developed to understand participants' experiences during the COVID-19 pandemic. Participants reported on two specific COVID-19 experiences: "Have you received results confirming that you have a diagnosis of COVID-19 by a public health official?"; and "Has somebody you know died after contracting the Coronavirus?". Response options were "yes" or "no". In addition, the well-established Perceived Stress Scale (PSS; Cohen et al., 1983) was slightly modified to measure perceived stress associated with, and/or specifically due to, the COVID-19 pandemic that occurred during the past week. The modified scale consisted of 10 items that were anchored to the coronavirus pandemic (e.g., "In the last week, how often have you felt nervous and stressed due to the coronavirus?'). Responses were recorded using the original 5-point Likert scale that ranged from 0 (never) to 4 (very often). The internal consistency of this scale ranged from $\alpha=.84-.89$ across time points in the present study.

\section{Anxiety}

Participants completed the Overall Anxiety Severity and Impairment Scale (OASIS; Norman et al., 2006). This self-report scale of anxiety contains five questions rated on a five-point Likert scale. Past psychometric work using receiver operating characteristic curves have demonstrated that a cutoff score of $\geq 8$ for the OASIS provides good sensitivity and specificity for identifying clinically significant levels of anxiety (Bentley et al., 2014; Campbell-Sills et al., 
2009; Norman et al., 2011). The internal consistency of this scale was very high, ranging from $\alpha=.90-.93$ across time points within the present study.

\section{Depression}

Participants also completed the Overall Depression Severity and Impairment Scale (ODSIS; Bentley et al., 2014). This self-report scale of depression contains five questions rated on a five-point Likert scale. Past psychometric work using receiver operating characteristic curves have demonstrated that cutoffs of $\geq 8$ for the ODSIS provide good sensitivity and specificity for identifying clinically significant levels of depression (Bentley et al., 2014; Campbell-Sills et al., 2009; Norman et al., 2011). The internal consistency of this scale was excellent, ranging from $\alpha=.94-.95$ across time points within the current study.

\section{Functional Impairment}

The survey included the Work and Social Adjustment Scale (WSAS; Mundt et al., 2002) to measure impairment in functioning. This self-report scale contains five questions assessing impairment across five domains: work, home management, social leisure activities, private leisure activities, and family and relationships. Level of impairment was rated from 0 (not at all) to 8 (very severely) for each item. The WSAS showed excellent internal consistency $(\alpha=.96-.97)$ across time points in the present study.

\section{Data Analysis}

Analyses consisted of effect sizes to quantify changes in outcomes within individuals across the four waves of data collection (standardized mean gain, ESsg with 95\% CI). ESsg can be interpreted in a manner similar to Cohen's d, but was more appropriate for quantifying intraindividual change in the present study as it includes a correction for associations between repeated measurements of outcomes (Lipsey \& Wilson, 2001). We then estimated the proportion of individuals who reported symptoms of anxiety or depression above clinical cutoffs indicating a probable anxiety or depressive disorder diagnosis based on the OASIS and ODSIS. Latent growth curve modeling (LGC) was then used to quantify intraindividual trajectories of change in continuous outcomes and to explore how demographic characteristics, COVID-19 experiences, and COVID-19 perceived stress predicted trajectories of change in outcomes. LGC were conducted using Mplus 8.0 (Muthén \& Muthén, 1998-2017) and robust maximum likelihood estimation. Second-order LGC models were specified such that each outcome was a latent variable at each timepoint with intercept and slope factors parameterized to estimate linear trajectories of change across the four waves with the intercept of all models set to time 1 . Second order LGC models were specified in order to improve the precision and statistical power of estimates in the LGC models by accounting for measurement error of outcomes at each time point (Little, 2013). The COVID-19 perceived stress latent variable was identified using three parcels that were created by randomly assigning items from the COVID-19 stress scale as indicators. Parcels are a modeling technique in which individual items are aggregated to create a smaller number to serve as indicators of a latent construct and have psychometric advantages (e.g., Little et al., 2002). The anxiety, depression, and functional impairment latent variables were identified using the five items from the respective scales as indicators. Unconditional LGC models were specified first to characterize inter and intraindividual differences in the trajectories of change in the four outcomes. Conditional LGC were then specified so that age (years) and dummy codes representing gender $(1=$ female, $0=$ male $)$, race $(1=$ White; $0=$ not White), report of confirmed COVID-19 diagnosis ( $1=$ yes, $0=$ no), and report of knowing an individual who died due to COVID-19 $(1=$ yes, $0=$ no) were included as covariates. For the conditional LGC, unstandardized and partially standardized results were estimated to aid in the interpretation of the magnitude of effects associated with demographic characteristics, COVID-19 experiences, and COVID-19 perceived stress. Model fit for the LGC analyses was evaluated using standard model fit indices and criteria, including: root-meansquare error of approximation (RMSEA; Steiger, 1990), the Tucker-Lewis index (TLI; Tucker \& Lewis, 1973), the comparative fit index (CFI; Bentler, 1990), and the standardized root-mean-square residual (SRMR; Jöreskog \& Sörbom, 1996). Acceptable model fit was evaluated using standard model fit criteria, including: RMSEA values below 0.08, and CFI and NNFI values above 0.95 (Hu \& Bentler, 1998).

\section{Results}

Bivariate correlations between main study variables are presented in Table 1 and descriptive results and withinperson effect sizes across the four waves of data collection are presented in Table 2. Intraindividual changes across the four waves appeared to be mostly consistent across the four outcomes (i.e., The largest decrease in outcomes consistently occurred between waves 1 and 2). Decreases in all four outcomes were statistically significant based on the confidence intervals of the effect sizes and all small to moderate effect size magnitude. Additional, but smaller decreases then happened between waves 2 and 3, as well as waves 3 and 4 for COVID stress, anxiety, and depression, with the overall decreases in these outcomes being moderate to large in effect size magnitude. Impairment decreased slightly 


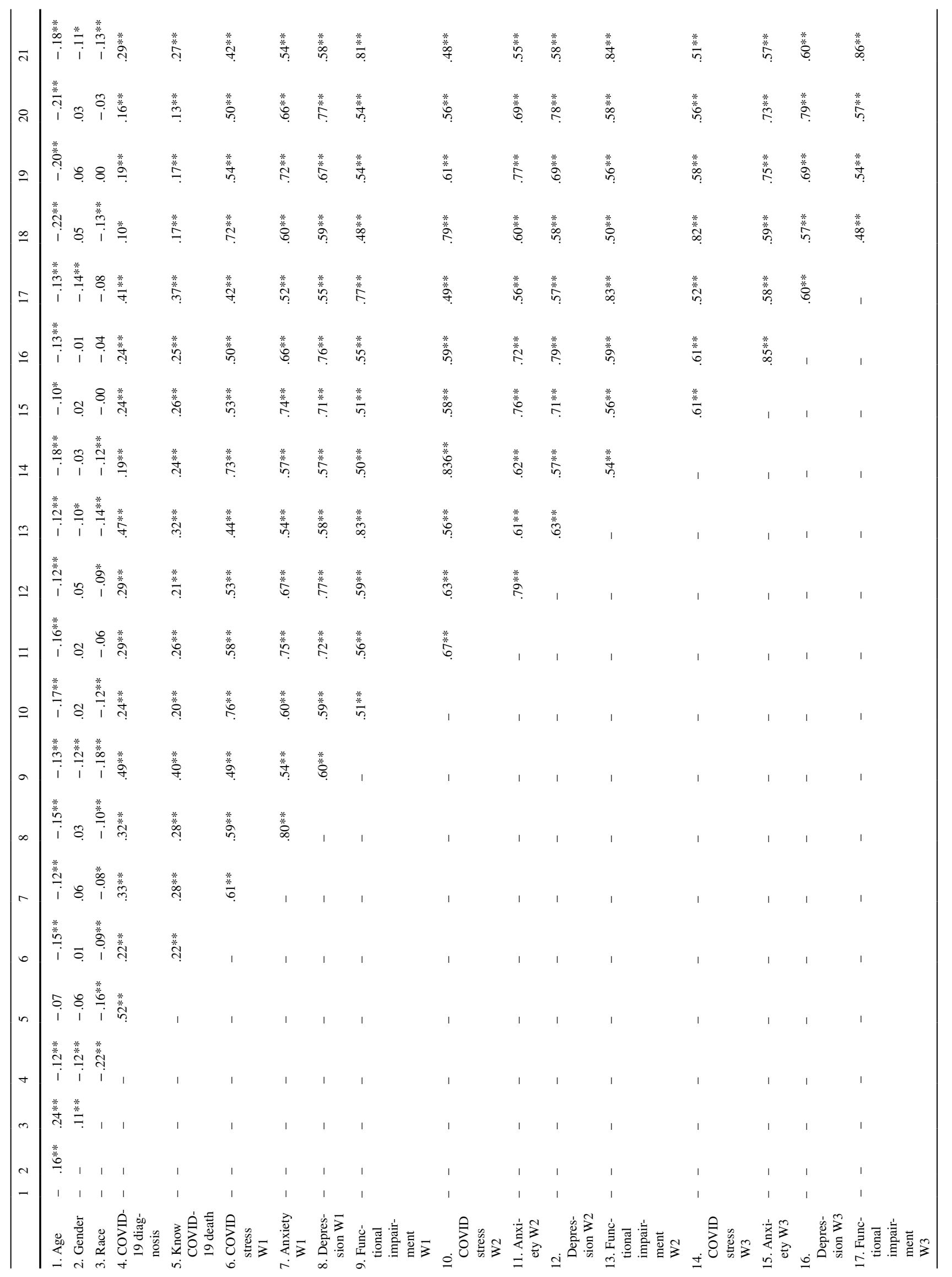




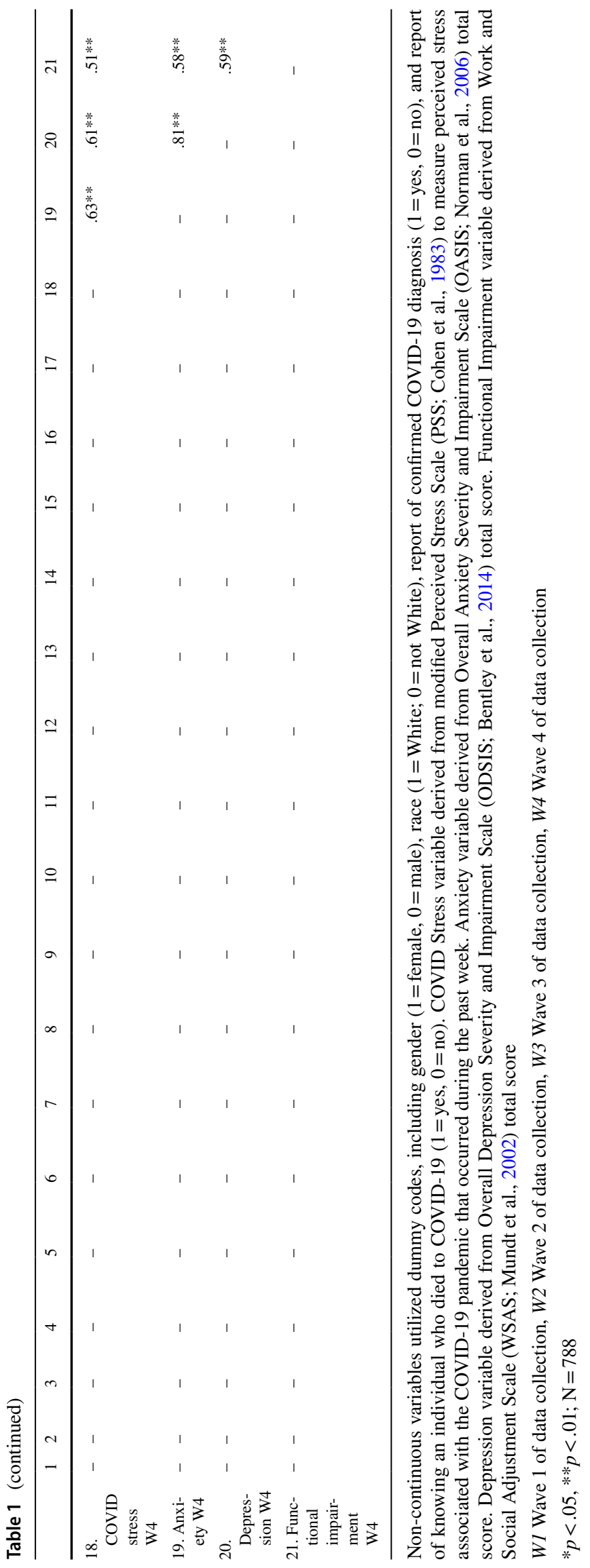


Table 2 Descriptives and within person effect sizes across four waves

\begin{tabular}{|c|c|c|c|c|c|c|c|}
\hline \multirow[t]{2}{*}{ Outcome } & \multicolumn{4}{|l|}{ Mean $(95 \%$ CI $)$} & \multicolumn{3}{|l|}{ ESsg $(95 \% \mathrm{CI})$} \\
\hline & W1 & W2 & W3 & W4 & W1-W2 & W1-W3 & W1-W4 \\
\hline COVID stress & $\begin{array}{l}16.58[16.05 \\
17.11]\end{array}$ & $\begin{array}{l}14.80[14.25 \\
15.36]\end{array}$ & $\begin{array}{c}13.90[13.35, \\
14.45]\end{array}$ & $\begin{array}{c}13.71[13.15 \\
14.26]\end{array}$ & $\begin{array}{l}-0.45 \\
\quad[-0.55,-0.35]\end{array}$ & $\begin{array}{l}-0.64 \\
\quad[-0.75,-0.54]\end{array}$ & $\begin{array}{l}-0.67 \\
\quad[-0.77,-0.56]\end{array}$ \\
\hline Anxiety & $5.86[5.52,6.20]$ & $4.70[4.41,4.99]$ & $4.09[3.81,4.38]$ & $3.93[3.65,4.22]$ & $\begin{array}{l}-0.46 \\
{[-0.56,-0.36]}\end{array}$ & $\begin{array}{l}-0.66 \\
{[-0.77,-0.56]}\end{array}$ & $\begin{array}{l}-0.67 \\
{[-0.70,-0.57]}\end{array}$ \\
\hline Depression & $4.75[4.40,5.10]$ & $3.89[3.58,4.20]$ & $3.37[3.08,3.66]$ & $3.11[2.82,3.39]$ & $\begin{array}{l}-0.33 \\
\quad[-0.43,-0.23]\end{array}$ & $\begin{array}{l}-0.52 \\
\quad[-0.62,-0.41]\end{array}$ & $\begin{array}{l}-0.58 \\
\quad[-0.68,-0.48]\end{array}$ \\
\hline $\begin{array}{l}\text { Functional impair- } \\
\text { ment }\end{array}$ & $\begin{array}{l}10.99[10.10, \\
11.89]\end{array}$ & $10.10[9.24,10.96]$ & $10.06[9.22,10.9]$ & $10.43[9.58,11.28]$ & $\begin{array}{l}-0.18 \\
\quad[-0.28,-0.08]\end{array}$ & $\begin{array}{l}-0.16 \\
\quad[-0.26,-0.06]\end{array}$ & $\begin{array}{l}-0.11 \\
\quad[-0.21,-0.01]\end{array}$ \\
\hline
\end{tabular}

COVID Stress variable derived from modified Perceived Stress Scale (PSS; Cohen et al., 1983) to measure perceived stress associated with the COVID-19 pandemic that occurred during the past week. Anxiety variable derived from Overall Anxiety Severity and Impairment Scale (OASIS; Norman et al., 2006) total score. Depression variable derived from Overall Depression Severity and Impairment Scale (ODSIS; Bentley et al., 2014) total score. Functional Impairment variable derived from Work and Social Adjustment Scale (WSAS; Mundt et al., 2002) total score

ESsg effect size standardized mean gain, $W$ wave

from wave 2 to wave 3 , but then increased from wave 3 to 4 and, while statistically significant, had a smaller overall decrease in effect size magnitude compared to the other three outcomes. These findings suggest a general decline in psychological distress associated with the COVID-19 pandemic from April/May 2020 to September/October 2020, but that the associated changes in functional impairment were only evident between wave 1 and 2 with no subsequent linear decrease.

The observed rates of probable anxiety or depressive disorder diagnosis based on the OASIS/ODSIS cutoffs were also indicative of a general decline in psychological distress across the four waves. The rates of probable anxiety disorder consistently decreased from wave $1(35.4 \%)$ to wave 2 $(21.3 \%)$ to wave $3(16.1 \%)$ to wave $4(15.6 \%)$. Similarly, the rates of probable depressive disorder consistently decreased from wave $1(29.6 \%)$ to wave $2(18.1 \%)$ to wave $3(14.5 \%)$ to wave $4(13.8 \%)$. These rates indicate that, while many individuals still reported moderate to high levels of anxiety and/or depression, the rates of clinically significant anxiety or depressive disorders decreased from the Spring to the Fall of 2020.

\section{Trajectories of Distress Outcomes}

We next examined intraindividual trajectories of change in these outcomes across the four waves using LGC models. Unconditional models were specified first to quantify change trajectories in the absence of any demographic or COVID19 experiences as predictors. The results of these models are presented in Table 3 and are consistent with the findings from the effect size analyses. Trajectories for COVID19 perceived stress, anxiety, and depression all indicated a decline in outcomes across the four waves whereas trajectories for functional impairment indicated minimal linear change across the four waves. A statistically significantly high level of variance in initial functional impairment scores was found, which may suggest that any differences in functional impairment across time were due to initial differences in impairment at the first wave of data collection. The associations between initial levels and trajectories of change were small in effect size magnitude and not statistically significant for any of the outcome measures.

\section{Predicting Trajectories of Distress and Impairment}

The final series of LGC models included age, gender, race, reported confirmed diagnosis of COVID, and report of knowing someone who passed away due to COVID-19 as predictors of both the intercepts and slopes of the four outcomes (see Tables 4, 5). Reporting a confirmed COVID19 diagnosis had the largest effect on the intercept of all outcomes and was consistently associated with greater levels of distress and impairment. Individuals who knew someone who passed away from COVID-19 also reported greater levels of distress and impairment, but the effects were only statistically significant for anxiety and impairment. Gender had the largest impact on the intercepts of anxiety and depression, with women reporting worse outcomes. Individuals identifying as White generally reported slightly less distress and impairment across outcomes, but none of the effects were statistically significant. Age had a consistent small, but statistically significant effect on the intercept of all outcomes such that older adults reported less distress and impairment. None of the predictors had a statistically significant impact on the slopes of outcomes, although a COVID-19 diagnosis and knowing someone that passed away were associated with small to moderate trends of greater distress and impairment for some outcomes. Together, these five variables predicted between approximately $11 \%$ and $30 \%$ of the variance $\left(R^{2}\right)$ in the 
Table 3 Individual trajectories in distress outcomes across four waves

\begin{tabular}{lllll}
\hline & Covid stress & Anxiety & Depression & Functional impairment \\
\hline $\begin{array}{lllll}\text { Model fit } \\
d f\end{array}$ & 50 & 166 & 166 & 166 \\
$\chi^{2}$ & 95.4 & 454.86 & 259.16 & 271.60 \\
RMSEA & .034 & .047 & .027 & .028 \\
CFI & .991 & .953 & .984 & .985 \\
TLI & .989 & .946 & .982 & .983 \\
Parameter estimates & & & & \\
Intercept mean (SE) & $0(0)$ & $0(0)$ & $0(0)$ & $0(0)$ \\
Intercept variance (SE) & $.374(.024)^{*}$ & $.668(.048)^{*}$ & $.869(.057)^{*}$ & $5.53(.28)^{*}$ \\
Slope mean (SE) & $-.073(.008)^{*}$ & $-.064(.010)^{*}$ & $-.042(.057)^{*}$ & $-.001(.022)$ \\
Slope variance (SE) & $.008(.003)^{*}$ & $.009(.005)$ & $.007(.007)$ & $.031(.030)$ \\
Intercept-slope correlation & $.076(.101)$ & $-.150(.129)$ & $-.134(.165)$ & $-.074(.135)$ \\
\hline
\end{tabular}

Model fit and estimates of temporal variation in covid-related stress, anxiety, depression, well-being, and functional impairment severity from latent growth curve models. COVID Stress variable derived from modified Perceived Stress Scale (PSS; Cohen et al., 1983) to measure perceived stress associated with the COVID-19 pandemic that occurred during the past week. Anxiety variable derived from Overall Anxiety Severity and Impairment Scale (OASIS; Norman et al., 2006) total score. Depression variable derived from Overall Depression Severity and Impairment Scale (ODSIS; Bentley et al., 2014) total score. Functional Impairment variable derived from Work and Social Adjustment Scale (WSAS; Mundt et al., 2002) total score

$S E$ standard error

$* p<.05$
Table 4 Model fit indices of conditional predicted trajectories of distress and impairment

\begin{tabular}{llllll}
\hline Outcome & $d f$ & $\chi^{2}$ & RMSEA & CFI & TLI \\
\hline Covid stress & 100 & 168.37 & .030 & .989 & .986 \\
Anxiety & 256 & 664.90 & .045 & .943 & .936 \\
Depression & 256 & 437.23 & .030 & .975 & .971 \\
Functional impairment & 256 & 403.42 & .027 & .983 & .981 \\
\hline
\end{tabular}

Model covariates included age, gender, race, report of confirmed COVID-19 diagnosis, and report of knowing an individual who passed away due to COVID-19

COVID Stress variable derived from modified Perceived Stress Scale (PSS; Cohen et al., 1983) to measure perceived stress associated with the COVID-19 pandemic that occurred during the past week. Anxiety variable derived from Overall Anxiety Severity and Impairment Scale (OASIS; Norman et al., 2006) total score. Depression variable derived from Overall Depression Severity and Impairment Scale (ODSIS; Bentley et al., 2014) total score. Functional Impairment variable derived from Work and Social Adjustment Scale (WSAS; Mundt et al., 2002) total score

$d f$ degrees of freedom, $\chi^{2}$ chi-square, RMSEA root-mean-square error of approximation, CFI comparative fit index, TLI Tucker-Lewis index, $S R M R$ standardized root-mean-square residual

intercept in outcomes and between approximately $6 \%$ and $9 \%$ of the variance in the slope of outcomes.

\section{Discussion}

As the world enters the second year of the COVID-19 pandemic, social distancing, constant media coverage, and COVID testing have become the "new normal". A growing body of research has documented and discussed sharp increases in mental health concerns at the outset of the pandemic (Czeisler et al., 2020; Gallagher et al., 2020; Hossain et al., 2020). The current study extends previous research by demonstrating that COVID-19 related stress, anxiety, and depression symptoms decreased over time. Over a period of 6 months (March-October 2020), effect size analyses and growth curve models demonstrated a substantial decrease in negative mental health outcomes, as well as rates of probable anxiety and depressive disorder diagnoses. A known COVID-19 diagnosis had the greatest impact on levels of negative mental health outcomes. In contrast to mental health outcomes, reports of functional impairment showed minimal to no change across the four waves.

These observed reductions in anxiety and depression are consistent with extant literature examining change in mental health outcomes after the 2009 H1N1influenza pandemic (Bults et al., 2015) and research examining anxiety and depression over a similar timeline in England (Fancourt et al., 2021). Our results support the presupposition that while the COVD-19 pandemic has caused harm to the population's overall mental health, individuals will begin to show resilience to impacts of the pandemic over time 
Table 5 Conditional predicted trajectories of distress and impairment by covariates

\begin{tabular}{|c|c|c|c|c|c|c|}
\hline \multirow[t]{2}{*}{ Outcome } & \multirow[t]{2}{*}{ Parameter } & \multicolumn{5}{|l|}{ Predictor } \\
\hline & & Age & Gender & Race & COVID-19 diagnosis & Know COVID-19 death \\
\hline \multicolumn{7}{|c|}{ Covid stress } \\
\hline \multirow[t]{3}{*}{ Intercept } & $B(\mathrm{SE})$ & $-.008 * * *(.002)$ & $.061(.048)$ & $-.046(.056)$ & $.367 * * *(.063)$ & $.112(.061)$ \\
\hline & $\beta(\mathrm{SE})$ & $-.013(.003)$ & $.099(.078)$ & $-.075(.091)$ & $.598(.103)$ & $.182(.100)$ \\
\hline & $R^{2}$ & .112 & - & - & - & - \\
\hline \multirow[t]{3}{*}{ Slope } & $B(\mathrm{SE})$ & $.000(.001)$ & $.001(.015)$ & $-.036(.019)$ & $.016(.023)$ & $.038(.020)$ \\
\hline & $\beta(\mathrm{SE})$ & $-.002(.006)$ & $.009(.166)$ & $-.386(.208)$ & $.168(.251)$ & $.413(.220)$ \\
\hline & $R^{2}$ & .092 & - & - & - & - \\
\hline \multicolumn{7}{|l|}{ Anxiety } \\
\hline \multirow[t]{3}{*}{ Intercept } & $B(\mathrm{SE})$ & $-.008 * * *(.003)$ & $.163 * *(.063)$ & $-.001(.073)$ & $.733 * * *(.099)$ & $.189 *(.080)$ \\
\hline & $\beta(\mathrm{SE})$ & $-.010(.003)$ & $.201(.077)$ & $-.001(.090)$ & $.905(.121)$ & $.233(.099)$ \\
\hline & $R^{2}$ & .190 & - & - & - & - \\
\hline \multirow[t]{3}{*}{ Slope } & $B(\mathrm{SE})$ & $-.001(.001)$ & $-.021(.020)$ & $.035(.025)$ & $-.041(.039)$ & $.024(.028)$ \\
\hline & $\beta(\mathrm{SE})$ & $-.007(.008)$ & $-.223(.212)$ & $.367(.257)$ & $-.436(.421)$ & $.249(.295)$ \\
\hline & $R^{2}$ & .061 & - & - & - & - \\
\hline \multicolumn{7}{|l|}{ Depression } \\
\hline \multirow[t]{3}{*}{ Intercept } & $B(\mathrm{SE})$ & $-.009 * *(.003)$ & $.162 *(.073)$ & $-.071(.083)$ & $.743 * * *(.108)$ & $.159(.088)$ \\
\hline & $\beta(\mathrm{SE})$ & $-.010(.003)$ & $.174(.078)$ & $-.076(.089)$ & $.799(.116)$ & $.171(.095)$ \\
\hline & $R^{2}$ & .151 & - & - & - & - \\
\hline \multirow[t]{3}{*}{ Slope } & $B(\mathrm{SE})$ & $.000(.001)$ & $-.034(.021)$ & $.043(.028)$ & $-.003(.043)$ & $-.010(.026)$ \\
\hline & $\beta(\mathrm{SE})$ & $.001(.100)$ & $-.403(.295)$ & $.500(.365)$ & $-.033(.509)$ & $-.112(.314)$ \\
\hline & $R^{2}$ & .086 & - & - & - & - \\
\hline \multicolumn{7}{|c|}{ Functional impairment } \\
\hline \multirow[t]{3}{*}{ Intercept } & $B(\mathrm{SE})$ & $-.012(.006)$ & $-.317 *(.154)$ & $-.341(.186)$ & $2.708 * * *(.270)$ & $.604 * *(.211)$ \\
\hline & $\beta(\mathrm{SE})$ & $-.005(.003)$ & $-.136(.066)$ & $-.146(.080)$ & $1.164(.113)$ & $.260(.091)$ \\
\hline & $R^{2}$ & .305 & - & - & - & - \\
\hline \multirow[t]{3}{*}{ Slope } & $B(\mathrm{SE})$ & $.000(.002)$ & $-.015(.042)$ & $.009(.047)$ & $.051(.068)$ & $.079(.053)$ \\
\hline & $\beta(\mathrm{SE})$ & $-.002(.009)$ & $-.082(.239)$ & $.048(.259)$ & $.283(.401)$ & $.438(.352)$ \\
\hline & $R^{2}$ & .071 & - & - & - & - \\
\hline
\end{tabular}

SE (standard error) presented in parentheses following beta parameter estimates. Models utilized dummy codes for non-continuous variables, including gender $(1=$ female, $0=$ male $)$, race $(1=$ White; $0=$ not White), report of confirmed COVID-19 diagnosis $(1=$ yes, $0=$ no), and report of knowing an individual who died to COVID-19 $(1=$ yes, $0=$ no). COVID Stress variable derived from modified Perceived Stress Scale (PSS; Cohen et al., 1983) to measure perceived stress associated with the COVID-19 pandemic that occurred during the past week. Anxiety variable derived from Overall Anxiety Severity and Impairment Scale (OASIS; Norman et al., 2006) total score. Depression variable derived from Overall Depression Severity and Impairment Scale (ODSIS; Bentley et al., 2014) total score. Functional Impairment variable derived from Work and Social Adjustment Scale (WSAS; Mundt et al., 2002) total score

$B$ unstandardized beta coefficient, $p$ probability value for unstandardized beta coefficient, $\beta$ standardized beta coefficient, $R^{2}$ standardized coefficient of determination

$* * * p<.001 ; * * p<.01 ; * p<.05$

(PeConga et al., 2020). The largest decrease in outcomes were between the first two waves, which supports theories on how individuals may respond to adverse life events. Bonnano et al., for instance, theorize that recovery is often characterized by a sharp increase in dysfunction followed by a gradual return to baseline levels of symptoms (Bonanno et al., 2011). Along these lines, O'Connor et al. (2020) found that levels of wellbeing increased while levels of anxiety, feelings of defeat decreased between March and May 2020. This trajectory of recovery appears to be comparable to the trajectories shown in the effect size analyses and unconditional LGC, particularly for COVID-19 related stress, anxiety, and depression outcomes.

The beginning of the United States' lockdown in midMarch 2020 was the context during which levels of stress, anxiety, depression, and impairment increased as individuals. In order to continue functioning in the face of adverse life events, such as those associated with the COVID-19 pandemic, one often develops coping strategies (Jaspal $\&$ Nerlich, 2020). The direction of the trajectories in the 
present study suggest that individuals may be using adaptive strategies such as anticipatory restructuring, in which individuals restructure their identity so that it is more in line with the environment (e.g. wearing masks; Jaspal \& Nerlich, 2020). Furthermore, increased familiarity with new habits and reduced pandemic-related uncertainty may have also contributed to lower levels of stress, anxiety and depression during the current study (Pedrozo-Pupo et al., 2020; Rettie \& Daniels, 2020).

While our findings demonstrated consistent decreases in the trajectory of COVID-19 related stress, anxiety, and depression, the pattern of change in functional impairment was unique. In addition to having the smallest degree of change over 6 months, functional impairment slightly increased between Wave 3 and Wave 4. The measure of functional impairment used in the current study (WSAS; Mundt et al., 2002) has been shown to be reliable, sensitive to change, and to measure factors distinct from anxiety and depression (Pedersen et al., 2017). Thus, our results indicated that while functional impairment may be a more chronic effect of pandemic stressors (Bauer et al., 2020), recovery trajectories for COVID-19 related stress, anxiety, and depression over time may reflect aspects of resiliency within the current study sample.

Trajectories of mental illness, stress, and impairment were not impacted by gender, race, confirmed diagnosis of COVID-19, or knowledge of someone dying due to COVID19. However, average outcome severity was influenced by these predictors. Individuals with a confirmed COVID-19 diagnosis, for example, demonstrated higher average levels of COVID-19 related stress, anxiety, depression, and functional impairment. The impact of age on distress and impairment is consistent with previous global findings showing that younger individuals reported more anxiety, depression, and stress compared to their older counterparts (Varma et al., 2020). As the mean age of the sample was approximately 37 years of age, we did not gain as much understanding of these outcome trajectories among elderly individuals, who may be particularly vulnerable due to isolation (Krendl \& Perry, 2021). Overall, these results underscore the value of providing mental health resources to those impacted by particular COVID-19 experiences.

\section{Limitations}

Despite a longitudinal study design and robust analytic technics, the current study was limited by the use of online self-report measurements. Therefore, effects due to fatigue or reporting bias must be considered while interpreting the results. To maximize validity, attention checks were used throughout the survey and data was examined after collection to search for complete invalid responses. Although we sampled from the U.S. population, the use of MTURK limits the generalizability of the sample, as there are specific qualifications (e.g. bank account, internet access) that are required in order to be an MTURK worker. The racial/ ethnic makeup of the sample, while comparable to the U.S. population based on census information, is not representative of major cities or areas of the United States where COVID-19 has had the greatest impact (e.g., New York City). Future research will improve the generalizability of the results through attempting to replicate these findings in a more racially, ethnically, and gender diverse samples. Future research may also benefit from gathering more specific information regarding geographic location (e.g., Northeast, Southwest), job type (e.g., healthcare worker, gig worker), and access to childcare as these factors have increasingly presented as relevant stressors for individuals and families over the course of the pandemic. Finally, these data were collected across four waves prior to the release of COVID-19 vaccines. Future studies may examine the trajectories of these outcomes among individuals after the vaccine became available to certain populations (e.g., healthcare workers, older adults).

\section{Conclusions}

The ongoing COVID-19 pandemic has raised concerns regarding mental health symptomatology and the current study responds to the call for research investigating longterm trajectories of psychiatric and functional impairment (Chen \& Bonanno, 2020). There is agreement across the literature that the COVID-19 pandemic has resulted in severe, adverse effects on the global population. In the United States, it appears that levels of perceived stress, anxiety, and depression improved over time. However, this change was not detected in functional impairment. The current study demonstrates support that while time may heal one's psychological well-being, it will take more than time to improve one's functioning. Therefore, our results highlight the importance of programs and assistance focused on improving one's work and social functioning.

\section{Declarations}

Conflict of interest Matthew W. Gallagher, Lia J. Smith, Angela L. Richardson, \& Laura J. Long declare that they have no conflict of interest.

Ethical Approval Ethics approval was obtained from the IRB of the University of Houston.

Research Involving Animal Rights No animal studies were carried out by the authors for this article. 
Informed Consent Informed consent was obtained from all individual participants included in the study.

\section{References}

Babore, A., Lombardi, L., Viceconti, M. L., Pignataro, S., Marino, V., Crudele, M., Candelori, C., Bramanti, S. M., \& Trumello, C. (2020). Psychological effects of the COVID-2019 pandemic: Perceived stress and coping strategies among healthcare professionals. Psychiatry Research, 293, 113366. https://doi.org/10.1016/j. psychres.2020.113366

Badola, M., Chauhan, R., Taneja, N., \& Janardhanan, R. (2021). A study on psychological distress, functional impairment, and social attitude toward the COVID-19 pandemic and lockdown among Indian population. Indian Journal of Social Psychiatry, 37(1), 64-70. https://doi.org/10.4103/ijsp.ijsp_192_20

Bauer, L., Broady, K., Edelberg, W., \& O'Donnell, J. (2020). Ten facts about COVID-19 and the U.S. economy. Brookings. Retrieved January 5, 2021, from https://www.brookings.edu/research/tenfacts-about-covid-19-and-the-u-s-economy/

Bendau, A., Petzold, M. B., Pyrkosch, L. M., Maricic, L., Betzler, F., Rogoll, J., Grobe, J., Ströhle, A., \& Plag, J. (2021). Associations between COVID-19 related media consumption and symptoms of anxiety, depression and COVID-19 related fear in the general population in Germany. European Archives of Psychiatry and Clinical Neuroscience, 271, 283-291. https://doi.org/10.1007/ s00406-020-01171-6

Bentler, P. M. (1990). Comparative fit indexes in structural models. Psychological Bulletin, 107(2), 238-246. https://doi.org/10.1037/ 0033-2909.107.2.238

Bentley, K. H., Gallagher, M. W., Carl, J. R., \& Barlow, D. H. (2014). Development and validation of the overall depression severity and impairment scale. Psychological Assessment, 26(3), 815-830. https://doi.org/10.1037/a0036216

Bonanno, G. A., Westphal, M., \& Mancini, A. D. (2011). Resilience to loss and potential trauma. Annual Review of Clinical Psychology, 7, 511-535. https://doi.org/10.1146/annurev-clinp sy-032210-104526

Breen, L. J., Lee, S. A., \& Neimeyer, R. A. (2021). Psychological risk factors of functional impairment after COVID-19 deaths. Journal of Pain and Symptom Management, 61(4), e1-e4. https://doi.org/ 10.1016/j.jpainsymman.2021.01.006

Bults, M., Beaujean, D. J., Richardus, J. H., \& Voeten, H. A. (2015). Perceptions and behavioral responses of the general public during the 2009 influenza A (H1N1) pandemic: A systematic review. Disaster Medicine and Public Health Preparedness, 9(2), 207-219. https://doi.org/10.1017/dmp.2014.160

Campbell-Sills, L., Norman, S. B., Craske, M. G., Sullivan, G., Lang, A. J., Chavira, D. A., Bystritsky, A., Sherbourne, C., Roy-Byrne, P., \& Stein, M. B. (2009). Validation of a brief measure of anxiety-related severity and impairment: The overall anxiety severity and impairment scale (OASIS). Journal of Affective Disorders, 112(1-3), 92-101. https://doi.org/10.1016/j.jad.2008.03.014

Chen, S., \& Bonanno, G. A. (2020). Psychological adjustment during the global outbreak of COVID-19: A resilience perspective. Psychological Trauma: Theory, Research, Practice and Policy, 12(S1), S51-S54. https://doi.org/10.1037/tra0000685

Chrousos, G. P. (2009). Stress and disorders of the stress system. Nature Reviews Endocrinology, 5, 374-381. https://doi.org/10. 1038/nrendo.2009.106

Cohen, S., Kamarck, T., \& Mermelstein, R. (1983). A global measure of perceived stress. Journal of Health and Social Behavior, 24(4), 385-396.
Czeisler, M. E., Lane, R. I., Petrosky, E., Wiley, J. F., Christensen, A., Njai, R., Weaver, M. D., Robbins, R., Facer-Childs, E. R., Barger, L. K., Czeisler, C. A., Howard, M. E., \& Rajaratnam, S. M. W. (2020). Mental health, substance use, and suicidal ideation during the COVID-19 pandemic-United States, June 24-30, 2020. Morbidity and Mortality Weekly Report, 69, 1049-1057. https:// doi.org/10.15585/mmwr.mm6932a1

Daly, M., Sutin, A. R., \& Robinson, E. (2020). Longitudinal changes in mental health and the COVID-19 pandemic: Evidence from the UK household longitudinal study. Psychological Medicine. https:// doi.org/10.1017/S0033291720004432

Das, A., Sil, A., Jaiswal, S., Rajeev, R., Thole, A., Jafferany, M., \& Ali, S. N. (2020). A study to evaluate depression and perceived stress among frontline Indian doctors combating the COVID-19 pandemic. Primary Care Companion for CNS Disorders. https:// doi.org/10.4088/PCC.20m02716

De France, K., Hancock, G. R., Stack, D. M., Serbin, L. A., \& Hollenstein, T. (2021). The mental health implications of COVID-19 for adolescents: Follow-up of a four-wave longitudinal study during the pandemic. The American Psychologist. https://doi.org/10. 1037/amp0000838

de Kloet, E. R., Joëls, M., \& Holsboer, F. (2005). Stress and the brain: From adaptation to disease. Nature Reviews: Neuroscience, 6(6), 463-475. https://doi.org/10.1038/nrn1683

Eisma, M. C., Boelen, P. A., \& Lenferink, L. I. M. (2020). Prolonged grief disorder following the Coronavirus (COVID-19) pandemic. Psychiatry Research, 288, 113031. https://doi.org/10.1016/j.psych res.2020.113031

Fancourt, D., Steptoe, A., \& Bu, F. (2021). Trajectories of anxiety and depressive symptoms during enforced isolation due to COVID-19 in England: A longitudinal observational study. The Lancet Psychiatry, 8(2), 141-149. https://doi.org/10.1016/S2215-0366(20) 30482-X

Gallagher, M. W., Zvolensky, M. J., Long, L. J., Rodgers, A. H., \& Garey, L. (2020). The impact of covid-19 experiences and associated stress on anxiety, depression, and functional impairment in American adults. Cognitive Therapy and Research, 44, 10431051. https://doi.org/10.1007/s10608-020-10143-y

Gruber, J., Prinstein, M. J., Clark, L. A., Rottenberg, J., Abramowitz, J. S., Albano, A. M., Aldao, A., Borelli, J. L., Chung, T., Davila, J., Forbes, E. E., Gee, D. G., Hall, G. C. N., Hallion, L. S., Hinshaw, S. P., Hofmann, S. G., Hollon, S. D., Joormann, J., Kazdin, A. E.,... Weinstock, L. M. (2020). Mental health and clinical psychological science in the time of COVID-19: Challenges, opportunities, and a call to action. The American Psychologist. 76, 409

Hickman, R. L., Jr., \& Douglas, S. L. (2010). Impact of chronic critical illness on the psychological outcomes of family members. $A A C N$ Advanced Critical Care, 21(1), 80-91. https://doi.org/10.1097/ NCI.0b013e3181c930a3

Hossain, M. M., Tasnim, S., Sultana, A., Faizah, F., Mazumder, H., Zou, L., McKyer, E. L. J., Ahmed, H. U., \& Ma, P. (2020). Epidemiology of mental health problems in COVID-19: A review. F1000Research, 9, 636. https://doi.org/10.12688/f1000research. 24457.1

Hu, L., \& Bentler, P. M. (1998). Fit indices in covariance structure modeling: Sensitivity to underparameterized model misspecification. Psychological Methods, 3(4), 424-453. https://doi.org/10. 1037/1082-989X.3.4.424

Jaspal, R., \& Nerlich, B. (2020). Social representations, identity threat, and coping amid COVID-19. Psychological Trauma: Theory, Research, Practice and Policy, 12(S1), S249-S251. https://doi. org/10.1037/tra0000773

Johns Hopkins University. (2021). COVID-19 dashboard by the Center for Systems Science and Engineering (CSSE) at Johns Hopkins University $(\mathrm{JHU})$. Johns Hopkins University \& Medicine Coronavirus Resource Center. 
Jöreskog, K. G., \& Sörbom, D. (1996). LISREL 8: User's reference guide. Scientific Software International.

Karlsson, L. C., Soveri, A., Lewandowsky, S., Karlsson, L., Karlsson, H., Nolvi, S., Karukivi, M., Lindfelt, M., \& Antfolk, J. (2021). Fearing the disease or the vaccine: The case of COVID-19. Personality and Individual Differences. https://doi.org/10.1016/j.paid. 2020.110590

Kim, H. F., Kunik, M. E., Molinari, V. A., Hillman, S. L., Lalani, S., Orengo, C. A., Petersen, N. J., Nahas, Z., \& Goodnight-White, S. (2000). Functional impairment in COPD patients: The impact of anxiety and depression. Psychosomatics, 41(6), 465-471. https:// doi.org/10.1176/appi.psy.41.6.465

Krendl, A. C., \& Perry, B. L. (2021). The impact of sheltering in place during the COVID-19 pandemic on older adults' social and mental well-being. Journals of Gerontology: Social Sciences, 76(2), e53-e58. https://doi.org/10.1093/geronb/gbaa110

Lee, E. H. (2012). Review of the psychometric evidence of the perceived stress scale. Asian Nursing Research, 6(4), 121-127. https://doi.org/10.1016/j.anr.2012.08.004

Li, Y., Zhao, J., Ma, Z., McReynolds, L. S., Lin, D., Chen, Z., Wang, T., Wang, D., Zhang, Y., Zhang, J., Fan, F., \& Liu, X. (2021). Mental health among college students during the COVID-19 pandemic in China: A 2-wave longitudinal survey. Journal of Affective Disorders, 281, 597-604. https://doi.org/10.1016/j.jad.2020.11.109

Little, T. D. (2013). Longitudinal structural equation modeling. Guilford Press.

Little, T. D., Cunningham, W. A., Shahar, G., \& Widaman, K. F. (2002). To parcel or not to parcel: Exploring the question, weighing the merits. Structural Equation Modeling: A Multidisciplinary Journal, 9(2), 151-173. https://doi.org/10.1207/S15328007S EM0902 1

Littlefield, C. H., Rodin, G. M., Murray, M. A., \& Craven, J. L. (1990). Influence of functional impairment and social support on depressive symptoms in persons with diabetes. Health Psychology, 9(6), 737-749. https://doi.org/10.1037//0278-6133.9.6.737

Lipsey, M. W., \& Wilson, D. B. (2001). Practical meta-analysis. SAGE Publications, Inc.

McEwen, B. S. (2005). Stressed or stressed out: What is the difference? Journal of Psychiatry and Neuroscience, 30(5), 315-318.

Moos, R. H., \& Schaefer, J. A. (1984). The crisis of physical illness. In R. H. Moos (Ed.), Coping with physical illness (pp. 3-25). Springer.

Mundt, J. C., Marks, I. M., Shear, M. K., \& Greist, J. H. (2002). The work and social adjustment scale: A simple measure of impairment in functioning. British Journal of Psychiatry, 180, 461-464. https://doi.org/10.1192/bjp.180.5.461

Muthén, L. K., \& Muthén, B. O. (1998-2017). Mplus User's Guide (8th ed.). Muthén \& Muthén, Berlin.

Norman, S. B., Campbell-Sills, L., Hitchcock, C. A., Sullivan, S., Rochlin, A., Wilkins, K. C., \& Stein, M. B. (2011). Psychometrics of a brief measure of anxiety to detect severity and impairment: The overall anxiety severity and impairment scale (OASIS). Journal of Psychiatric Research, 45(2), 262-268. https://doi.org/10. 1016/j.jpsychires.2010.06.011

Norman, S. B., Cissell, S. H., Means-Christensen, A. J., \& Stein, M. B. (2006). Development and validation of an overall anxiety severity and impairment scale (OASIS). Depression and Anxiety, 23(4), 245-249. https://doi.org/10.1002/da.20182

Nyer, M., Farabaugh, A., Fehling, K., Soskin, D., Holt, D., Papakostas, G. I., Pedrelli, P., Fava, M., Pisoni, A., Vitolo, O., \& Mischoulon, D. (2013). Relationship between sleep disturbance and depression, anxiety, and functioning in college students. Depression and Anxiety, 30(9), 873-880. https://doi.org/10.1002/da.22064

O'Connor, R. C., Wetherall, K., Cleare, S., McClelland, H., Melson, A. J., Niedzwiedz, C. L., O'Carroll, R. E., O'Connor, D. B., Platt, S., Scowcroft, E., Watson, B., Zortea, T., Ferguson, E., \&
Robb, K. A. (2020). Mental health and well-being during the COVID-19 pandemic: Longitudinal analyses of adults in the UK COVID-19 Mental Health \& Wellbeing study. The British Journal of Psychiatry. https://doi.org/10.1192/bjp.2020.212

Ott, C. H. (2003). The impact of complicated grief on mental and physical health at various points in the bereavement process. Death Studies, 27(3), 249-272. https://doi.org/10.1080/07481 180302887

PeConga, E. K., Gauthier, G. M., Holloway, A., Walker, R. S. W., Rosencrans, P. L., Zoellner, L. A., \& Bedard-Gilligan, M. (2020). Resilience is spreading: Mental health within the COVID-19 pandemic. Psychological Trauma: Theory, Research, Practice and Policy, 12(S1), S47-S48. https://doi.org/10.1037/tra0000874

Pedersen, G., Kvarstein, E. H., \& Wilberg, T. (2017). The work and social adjustment scale: Psychometric properties and validity among males and females, and outpatients with and without personality disorders. Personality and Mental Health, 11(4), 215228. https://doi.org/10.1002/pmh.1382

Pedrozo-Pupo, J. C., Pedrozo-Cortés, M. J., \& Campo-Arias, A. (2020). Perceived stress associated with COVID-19 epidemic in Colombia: An online survey. Cadernos De Saude Publica, 36(5), e00090520. https://doi.org/10.1590/0102-311x00090520

Pfefferbaum, B., \& North, C. S. (2020). Mental health and the COVID19 pandemic. New England Journal of Medicine, 383(6), 510 512. https://doi.org/10.1056/NEJMp2008017

Pierce, M., Hope, H., Ford, T., Hatch, S., Hotopf, M., John, A., Kontopantelis, E., Webb, R., Wessely, S., McManus, S., \& Abel, K. M. (2020). Mental health before and during the COVID-19 pandemic: A longitudinal probability sample survey of the UK population. The Lancet Psychiatry, 7(10), 883-892. https://doi.org/10.1016/ S2215-0366(20)30308-4

Redmond, N., Richman, J., Gamboa, C. M., Albert, M. A., Sims, M., Durant, R. W., Glasser, S. P., \& Safford, M. M. (2013). Perceived stress is associated with incident coronary heart disease and allcause mortality in low- but not high-income participants in the reasons for geographic and racial differences in stroke study. Journal of the American Heart Association, 2(6), e000447. https://doi. org/10.1161/jaha.113.000447

Rettie, H., \& Daniels, J. (2020). Coping and tolerance of uncertainty: Predictors and mediators of mental health during the COVID-19 pandemic. The American Psychologist. https://doi.org/10.1037/ amp0000710

Steiger, J. H. (1990). Structural model evaluation and modification: An interval estimation approach. Multivariate Behavioral Research, 25(2), 173-180. https://doi.org/10.1207/s15327906mbr2502_4

Taquet, M., Geddes, J. R., Husain, M., Luciano, S., \& Harrison, P. J. (2021). 6-month neurological and psychiatric outcomes in 236,379 survivors of COVID-19: A retrospective cohort study using electronic health records. Lancet Psychiatry, 8(5), 416-427. https://doi.org/10.1016/s2215-0366(21)00084-5

Thomas, K. A., \& Clifford, S. (2017). Validity and mechanical turk: An assessment of exclusion methods and interactive experiments. Computers in Human Behavior, 77, 184-197.

Tucker, L. R., \& Lewis, C. (1973). A reliability coefficient for maximum likelihood factor analysis. Psychometrika, 38, 1-10. https:// doi.org/10.1007/BF02291170

Turner, A., \& Baker, A. L. (2010). The psychological impact of serious illness. In D. B. Cooper (Ed.), Introduction to mental health substance use (pp. 94-107). Radcliffe.

Umucu, E., \& Lee, B. (2020). Examining the impact of COVID-19 on stress and coping strategies in individuals with disabilities and chronic conditions. Rehabilitation Psychology, 65(3), 193-198. https://doi.org/10.1037/rep0000328

Varma, P., Junge, M., Meaklim, H., \& Jackson, M. L. (2020). Younger people are more vulnerable to stress, anxiety and depression during COVID-19 pandemic: A global cross-sectional survey. 
Progress in Neuro-Psychopharmacology and Biological Psychiatry, 109, 110236. https://doi.org/10.1016/j.pnpbp.2020.110236

Wang, C., Pan, R., Wan, X., Tan, Y., Xu, L., Ho, C. S., \& Ho, R. C. (2020a). Immediate psychological responses and associated factors during the initial stage of the 2019 coronavirus disease (COVID-19) epidemic among the general population in China. International Journal of Environmental Research and Public Health. https://doi.org/10.3390/ijerph17051729

Wang, C., Pan, R., Wan, X., Tan, Y., Xu, L., McIntyre, R. S., Choo, F. N., Tran, B., Ho, R., Sharma, V. K., \& Ho, C. (2020b). A longitudinal study on the mental health of general population during the
COVID-19 epidemic in China. Brain, Behavior, and Immunity, 87, 40-48. https://doi.org/10.1016/j.bbi.2020.04.028

Yao, H., Chen, J. H., \& Xu, Y. F. (2020). Patients with mental health disorders in the COVID-19 epidemic. Lancet Psychiatry, 7(4), e21. https://doi.org/10.1016/s2215-0366(20)30090-0

Publisher's Note Springer Nature remains neutral with regard to jurisdictional claims in published maps and institutional affiliations. 